\title{
Multi-Component and Multi-Point Trace Gas Sensing in Wavelength Modulation Spectroscopy Based on Wavelength Stabilization
}

\author{
Zongliang $\mathrm{WANG}^{1 *}$, Jun $\mathrm{CHANG}^{2}$, Huishan $\mathrm{YU}^{1}$, Cunwei TIAN ${ }^{1}$, Hao ZHANG ${ }^{1}$, \\ Xiukun ZHANG ${ }^{1}$, Longfei TANG ${ }^{1}$, Qinduan $\mathrm{ZHANG}^{2}$, and Yiwen $\mathrm{FENG}^{2}$ \\ ${ }^{1}$ School of Physics Science and Information Technology and Shandong Key Laboratory of Optical Communication \\ Science and Technology, Liaocheng University, Liaocheng 252000, China \\ ${ }^{2}$ School of Information Science and Engineering and Shandong Provincial Key Laboratory of Laser Technology and \\ Application, Shandong University, Jinan 250100, China
}

${ }^{*}$ Corresponding author: Zongliang WANG E-mail: wangzongliang@lcu.edu.cn

\begin{abstract}
Multi-component and multi-point trace gas sensing in the wavelength modulation spectroscopy is demonstrated based on the frequency-division multiplexing and time-division multiplexing technology. A reference photodetector is connected in series with a reference gas cell with the constant concentration to measure the second-harmonics peak of the components for wavelength stabilization in real time. The central wavelengths of the distributed feedback lasers are locked to the target gas absorption centers by the reference second-harmonics signal using a digital proportional-integral-derivative controller. The distributed feedback lasers with different wavelengths and modulation frequencies are injected into the gas cell to achieve multi-components gas measurement by the frequency-division multiplexing technology. In addition, multi-point trace gas sensing is achieved by the time-division multiplexing technology using a photoswitch and a relay unit. We use this scheme to detect methane $\left(\mathrm{CH}_{4}\right)$ at $1650.9 \mathrm{~nm}$ and water vapor $\left(\mathrm{H}_{2} \mathrm{O}\right)$ at $1368.597 \mathrm{~nm}$ as a proof of principle with the gas cell path length of $10 \mathrm{~cm}$. The minimum detection limits achieved for $\mathrm{H}_{2} \mathrm{O}$ and $\mathrm{CH}_{4}$ are $1.13 \mathrm{ppm}$ and $11.85 \mathrm{ppm}$ respectively, with three-point gas cell measurement; thus 10.5-fold and 10.1-fold improvements are achieved in comparison with the traditional wavelength modulation spectroscopy. Meanwhile, their excellent $R$-square values reach 0.9983 and 0.99564 for the concentration ranges of $500 \mathrm{ppm}$ to $2000 \mathrm{ppm}$ and $800 \mathrm{ppm}$ to $2700 \mathrm{ppm}$, respectively.
\end{abstract}

Keywords: Wavelength modulation spectroscopy; wavelength stabilization; multi-point; multi-component; trace gas sensing.

Citation: Zongliang WANG, Jun CHANG, Huishan YU, Cunwei TIAN, Hao ZHANG, Xiukun ZHANG, et al., "Multi-Component and Multi-Point Trace Gas Sensing in Wavelength Modulation Spectroscopy Based on Wavelength Stabilization," Photonic Sensors, 2019, 9(4): 376-387.

\section{Introduction}

Over the last few decades, tunable diode laser absorption spectroscopy (TDLAS) has been widely used in the trace gas detection in various fields, such as industrial production control [1], mine safety monitoring [2, 3], combustion processes $[4,5]$,

Received: 28 January 2019 / Revised 20 March 2019

(C) The Author(s) 2019. This article is published with open access at Springerlink.com

DOI: $10.1007 / \mathrm{s} 13320-019-0544-y$

Article type: Regular 
environmental monitoring [6, 7], and explosive analysis. Moreover, owing to the well-known advantages of resistance to electromagnetic interference, low cost, and portability, TDLAS has been widely studied [8, 9]. Among TDLAS techniques, the method that is often referred to as wavelength modulation spectroscopy (WMS) [10-12] is the most representative because of its high minimum detection limit (MDL) and sensitivity. In WMS, the gas concentration is deduced from the harmonics using a distributed feedback (DFB) laser modulated at a higher frequency of several $\mathrm{kHz}$. Then, a higher signal-to-noise ratio (SNR) can be achieved due to the suppression of $1 / f$ noise by the lock-in amplifier (LIA). WMS is typically used to detect trace gas because of its higher detection sensitivity in comparison with direct absorption spectroscopy.

With advances in science and technology, the application field of gas detection is expanding, and the number of gas detection sensors is also increasing. To reduce the examination costs, multi-component and multi-point gas sensors have been intensively studied and have attracted much attention. In multi-point gas sensor research, Eich et al. proposed a multi-point oxygen sensing based on optical time-domain reflectometry and the luminescence quenching of a sensor dye. They achieved two-sensor-point measurement by using 90-m-long $200 / 220$ quartz / quartz fibers, a laser with an excitation wavelength of $355 \mathrm{~nm}$ and a $2.5 \mathrm{kHz}$ repetition rate (pulse width: $5 \mathrm{~ns}$; pulse energy: $25 \mu \mathrm{J}$ ), and a sensor dye with a decay time of $80 \mathrm{~ns}[13,14]$. Sun et al. proposed a multi-point remote methane measurement system based on spectrum absorption and reflective time-domain multiplexing. The concentration and position information is simultaneously obtained by the optimized gas chamber with the reflective mode. There are three measurement points along a $4 \mathrm{~km}$ optical length in the experiment. A $2 \% \mathrm{MDL}$ reduction is achieved, and the linear correlation coefficients are $0.999,0.996$, and 0.989 at $0 \mathrm{~km}$, $2 \mathrm{~km}$, and $4 \mathrm{~km}$, respectively [15]. Liu et al. proposed and demonstrated a multi-channel fiber surface plasmon resonance sensor based on time-division multiplexing (TDM) by using the multi-core fiber. The multiple cores are multiple sensing zones, which are equivalent to subdivision of the traditional single-fiber core into multiple independent sensing zones, realizing multi-channel surface plasmon resonance sensing. By combining TDM and wavelength-division multiplexing (WDM), the sensing channels can be doubled [16]. Yu et al. proposed a fiber optical multi-point acetylene sensing system using dense WDM, which took advantage of different absorption lines to tag different probes in the system. Ma et al. demonstrated long-distance distributed gas sensing by using a micro-nanofiber evanescent wave quartz-enhanced photoacoustic spectroscopy technique. A $3 \mathrm{~km}$ single-mode fiber with three tapers and an erbium-doped fiber amplifier with an output optical power of $700 \mathrm{~mW}$ were employed, and the MDLs of the three tapers were $30 \mathrm{ppm}$, $51 \mathrm{ppm}$, and $13 \mathrm{ppm}$, respectively [17].

In multi-component gas sensor research, Yu et al. used WDM to realize multiband laser operation for multi-gas detection. The system is applied to detect mixtures of acetylene, carbon monoxide, and carbon dioxide in the $\mathrm{C}+\mathrm{L}$ band, and the minimum detectable concentrations were $0.6 \mathrm{ppm}, 17.4 \mathrm{ppm}$, and $19.2 \mathrm{ppm}$, respectively [18]. Wu et al. demonstrated a selective and sensitive quartz-enhanced photoacoustic spectroscopy (QEPAS) sensor, employing an erbium-doped fiber amplifier and a DFB laser operating at $1582 \mathrm{~nm}$ for the simultaneous detection of ammonia and hydrogen sulfide. The detection sensitivities $(1 \sigma)$ of 52 parts per billion by volume (ppbv) and $17 \mathrm{ppbv}$ for $\mathrm{NH}_{3}$ and $\mathrm{H}_{2} \mathrm{~S}$, respectively, were achieved [19]. Scherer et al. described a new mid-infrared sensor platform, which combined different frequency generation-based tunable laser sources with simple 
direct absorption spectroscopy. The sensors were capable of sub-ppb detection of key greenhouse gas species as well as common pollutants and tracer species [20]. Jahjah et al. developed a sensitive, selective, and well-established technique of QEPAS for $\mathrm{CH}_{4}$ and $\mathrm{N}_{2} \mathrm{O}$ measurements using a distributed-feedback quantum-cascade laser (DFB-QCL) in the $7.83 \mathrm{~mm}$ wavelength range. For the targeted $\mathrm{CH}_{4}$ and $\mathrm{N}_{2} \mathrm{O}$ absorption lines located at $1275.04 \mathrm{~cm}^{-1}$ and $1275.49 \mathrm{~cm}^{-1}$, detection limits of $13 \mathrm{ppbv}$ and 6 ppbv, respectively, were achieved with a $1 \mathrm{~s}$ data acquisition time [21]. Ma et al. demonstrated an ultra-sensitive and selective QEPAS sensor platform for the detection of $\mathrm{CO}$ and nitrous oxide $\mathrm{N}_{2} \mathrm{O}$ using a state-of-the-art $4.61 \mu \mathrm{m}$ high-power, continuous-wave DFB-QCL. The MDLs of $1.5 \mathrm{ppbv}$ and $23 \mathrm{ppbv}$ were obtained for the $\mathrm{CO}$ and $\mathrm{N}_{2} \mathrm{O}$ detection, respectively [22].

In addition to the above researches, some sensors have been introduced that are capable of both multi-component and multi-point gas detections. Zhang et al. demonstrated a novel intra-cavity fiber laser system that used fiber Bragg gratings as wavelength-selective cavity mirrors and a tunable filter to tune the operating wavelength to the Bragg wavelength of a selected Bragg grating, allowing the gas concentrations of multiple components at multiple locations to be determined [23]. Li applied frequency-shifted interferometry to acquire multiple gas sensors along a single fiber. This method uses a tunable continuous-wave laser and a slow detector, and allows a spectral overlap of sensors. It can be used to quantify the concentrations of single or multiple gas species at multiple locations [24]. Whitenett reported an initial study on the operation of a mode-locked fiber laser system for application in gas spectroscopy as a multi-point multi-gas sensor. Wavelength selection is performed by multiple chirped gratings, and fine tuning is based on the dispersion properties of the chirped gratings [25].

The above mentioned sensors are not very stable because the wavelength selection uses multiple chirped gratings or fiber Bragg gratings. In this study, multi-component and multi-point trace gas sensing in WMS is demonstrated based on the FDM and TDM, allowing a good stability. Meanwhile, a reference photodetector is connected in series with a reference gas cell of constant concentration to measure the $2 f$ peak of the multi-component for wavelength stabilization in real time. The central wavelengths of the DFB lasers are locked to the target gas absorption centers by the $2 f$ signal of the reference gas cell using a digital proportion integration differentiation (PID) controller. DFB lasers with various wavelengths and modulation frequencies are injected into the gas cell to achieve multi-component gas measurement by FDM. Multi-point trace gas sensing is achieved by TDM using the photoswitch and relay unit. We use this scheme to detect $\mathrm{CH}_{4}$ at $1650.9 \mathrm{~nm}$ [26] and $\mathrm{H}_{2} \mathrm{O}$ at $1368.597 \mathrm{~nm}$ as a proof of principle with a $10 \mathrm{~cm}$ gas cell path length, achieving a remarkable MDL reduction. To validate the feasibility of the proposed scheme, experiments are performed.

\section{Experiments}

\subsection{Experimental scheme}

\subsubsection{Multi-component and multi-point trace gas sensing scheme}

Multi-component and multi-point trace gas sensing is achieved by FDM and TDM. Every component has a unique modulation frequency and a laser wavelength corresponding to the gas absorption center, whose gas concentration is induced from the corresponding $2 f$ signals detected by the LIA. Multi-point trace gas sensing is achieved by TDM by using the photoswitch and relay unit. Different gas sensing points are detected at different times. The detailed method is shown in Fig. 1. Various modulation frequencies, $\omega_{1}, \omega_{2}$, $\omega_{3}, \cdots, \omega_{n}$, are used to drive the laser whose 
wavelengths, corresponding to the absorption wavelengths of each component gas. The second-harmonic signals, $2 \omega_{1}, 2 \omega_{2}, 2 \omega_{3}, \cdots, 2 \omega_{n}$, are excited by the gas absorption in the gas cell. The second-harmonic signals are detected by the LIA at the specific frequency of each component to calculate the gas concentration of Gas-1, Gas-2, Gas-3, $\cdots$, Gas- $n$. The gas concentrations of Point-1, Point- $2, \cdots$, Point $-n$ are determined by TDM by using the photoswitch and relay unit.

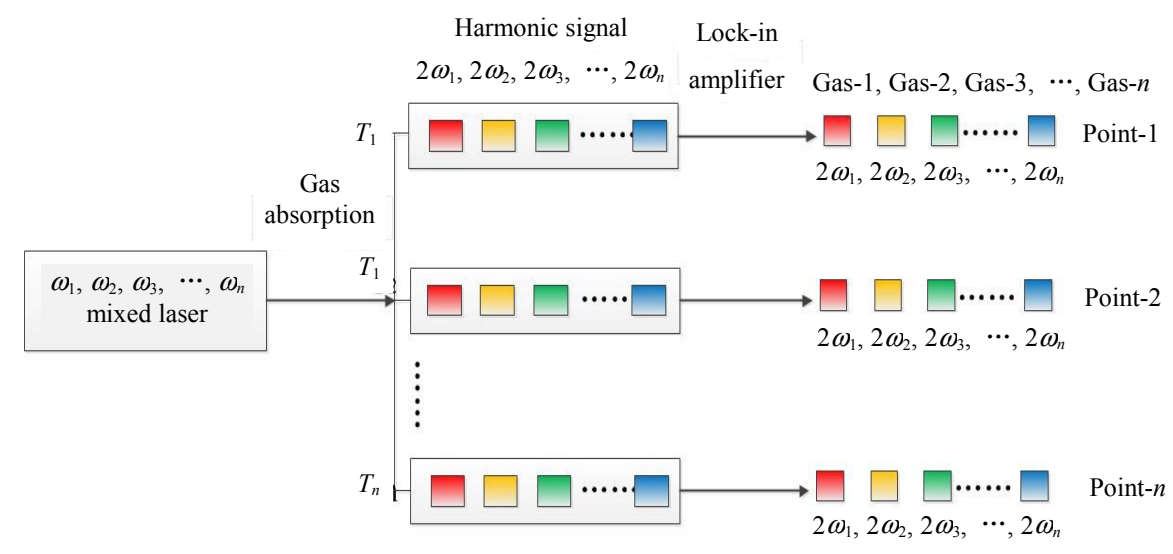

Fig. 1 Multi-component and multi-point trace gas sensing principle.

\subsubsection{Wavelength stabilization scheme}

A reference photodetector is connected in series with a reference gas cell with the constant concentration to detect the $2 f$ peak for wavelength stabilization in real time. The central wavelengths of the diode lasers are locked to the target gas absorption centers by the reference $2 f$ peak by using a digital PID controller. This method is described in details as follows:

(1) A signal combining sawtooth signal and modulation signal is used to drive the laser source, and the emitted light is propagated through the reference gas cell, exciting the second-harmonic signal because of gas absorption. Then, the laser is converted to an electrical signal by the photodetector connected to the reference gas cell and then is acquired by the data acquisition card (DAQ).

(2) The $2 f$ signal peak is extracted from the signal, which is transmitted to the computer, and then the wavelength driver current corresponding to the $2 f$ signal peak is defined as the one at the gas absorption center.
(3) The obtained current is set to the laser driver, and the gas sensing starts using the above constant driver current combined with a high-frequency sine modulation signal, which is used to excite the $2 f$ signal. Taking the second-harmonic peak of the reference gas cell as a reference, the central wavelengths of the DFB laser are locked to the target gas absorption centers using a digital PID controller in real time. The wavelength stabilization principle is shown in Fig. 2.

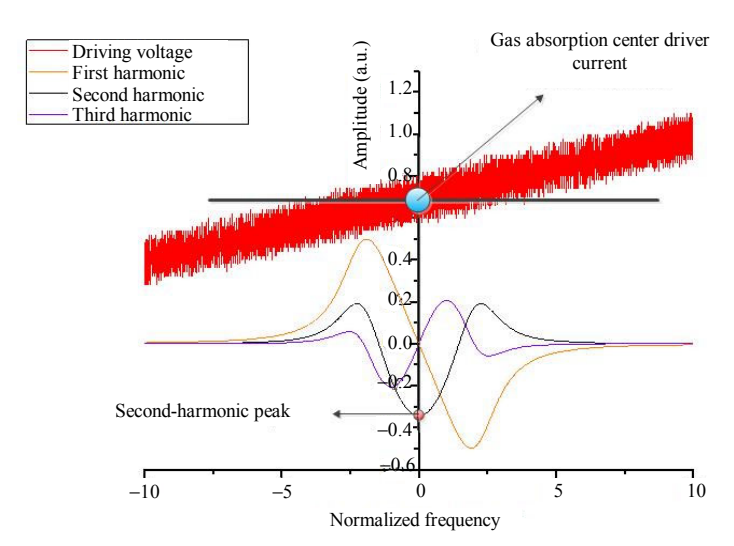

Fig. 2 First (1f), second (2f), and third (3f) harmonics and the driver signal of the DFB. 


\subsubsection{Signal processing in the system}

Taking two-component and three-point gas detection as an example, signal processing is realized on a computer, and its function diagram is shown in Fig. 3. The signal processing is implemented through two functional zones: the reference and the detection modules. In the reference part, the harmonics detected by the photodetector is extracted by the reference LIA and then is acquired by the DAQ for wavelength stabilization in the system by using the DFB laser with the low-frequency sawtooth and high-frequency sine wave modulation. The frequencies of the $2 f$ signal corresponding to the two components are expressed as $2 f_{1}$ and $2 f_{2}$. The peaks of the $2 f_{1}$ and $2 f_{2}$ signals are refined as set value-1 and set value- 2 at the nominal central wavelength. Then, the DFB lasers are modulated by the signal combined sine wave modulation with the driver current at set value- 1 and set value- 2 . The measured $2 f_{1}$ and $2 f_{2}$ are used as error signals for wavelength stabilization in real time. The current offset is iteratively revised by the PID module to make the $2 f$ signal closer to the set value. In particular, set value-1 and set value-2, shown in Fig. 3, must be characterized by scanning the laser frequency across the absorption profile. In the gas detection part, the $2 f_{1}$ and $2 f_{2}$ values collected by the photodetector in each gas cell are extracted by the LIA in turn by using the relay unit. Finally, $2 f_{1}$ and $2 f_{2}$ are acquired by the DAQ for the calculation of gas absorption.

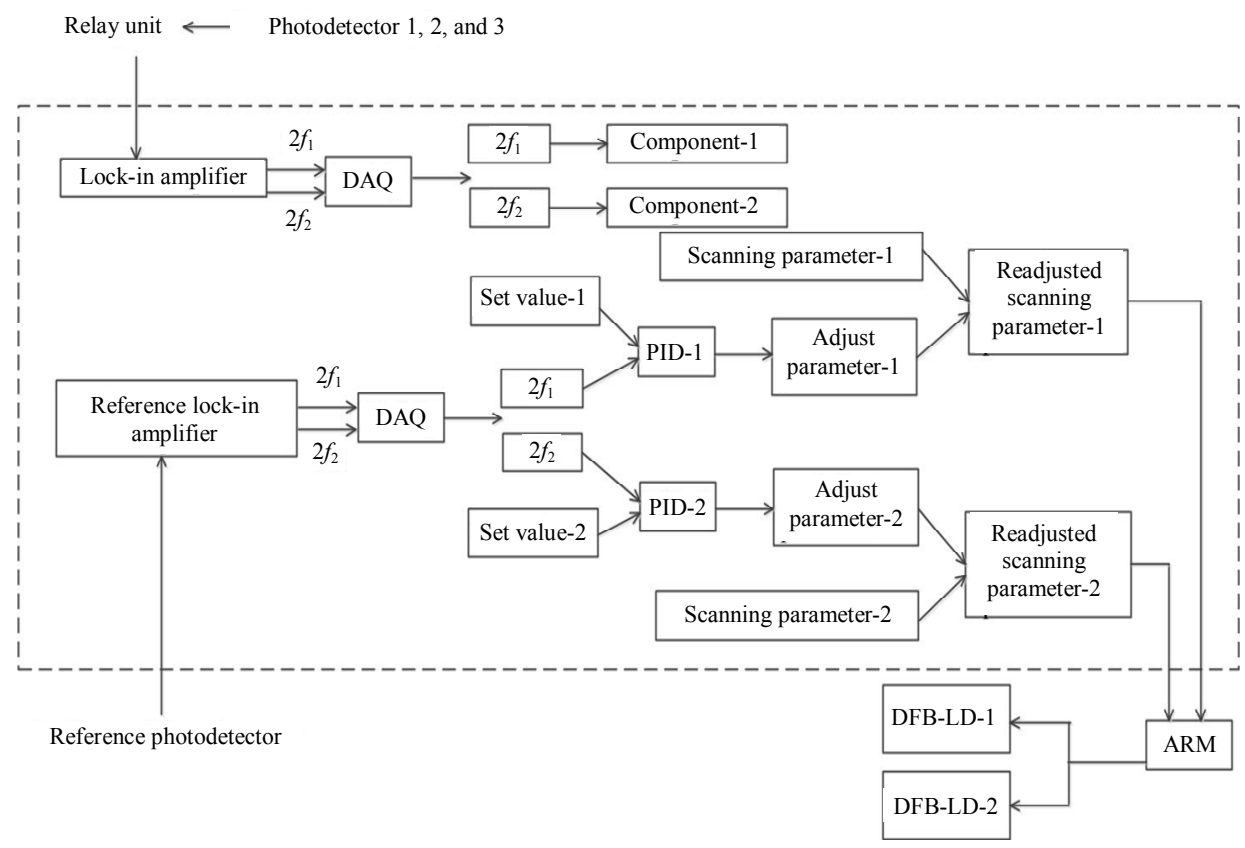

Fig. 3 Signal processing block diagram in the system.

\subsection{Experimental setup}

In the experiment, methane and water vapor detection at three points is conducted to verify the feasibility of the multi-component and multi-point trace gas sensing technique. The experimental setup is constructed as shown in Fig. 4. Methane and water vapor have strong absorption lines at $1650.9 \mathrm{~nm}$ and
$1368.597 \mathrm{~nm}$, respectively, according to HITRAN 2008, so 14-pin butterfly-packaged DFB lasers with central wavelengths of $1653.7 \mathrm{~nm}$ and $1368.30 \mathrm{~nm}$ (Wuhan 69 Sensor Technology, CHINA) are utilized as laser sources. An ARM7 (LPC1758, NXP, Netherlands) and a signal generator (FY2300A, Feel Tech, China) comprise a driving module to control 
the two DFB lasers. A combination of a sawtooth wave signal and a high-frequency modulation signal generated by the ARM7 and the signal generator, respectively, is used to drive the DFB laser. The two optical paths are divided by the $2 \times 2$ optical fiber coupler, responsible for reference and detection tasks. In the reference path, the $10 \mathrm{~cm}$ reference cell is connected to the coupler, inducing gas absorption. Then, the reference photodetector and LIA are used to collect and extract the $2 f$ signal for further processing. The output signal of the LIA is acquired by the DAQ for wavelength stabilization. The driver current at the gas absorption center obtained by the computer is transmitted to the ARM7 to complete the wavelength stabilization. In the detection path, a photoswitch is connected to the coupler to switch the three-gas cell in turn using TDM. The laser passing through the gas cell is collected by the photodetector for photoelectric conversion and then is transmitted to the LIA in turn by the relay unit for extraction of the $2 f$ signal. A DAQ is connected to the LIA for acquisition of the $2 f$ signal and transmission to the computer for gas absorption calculation.

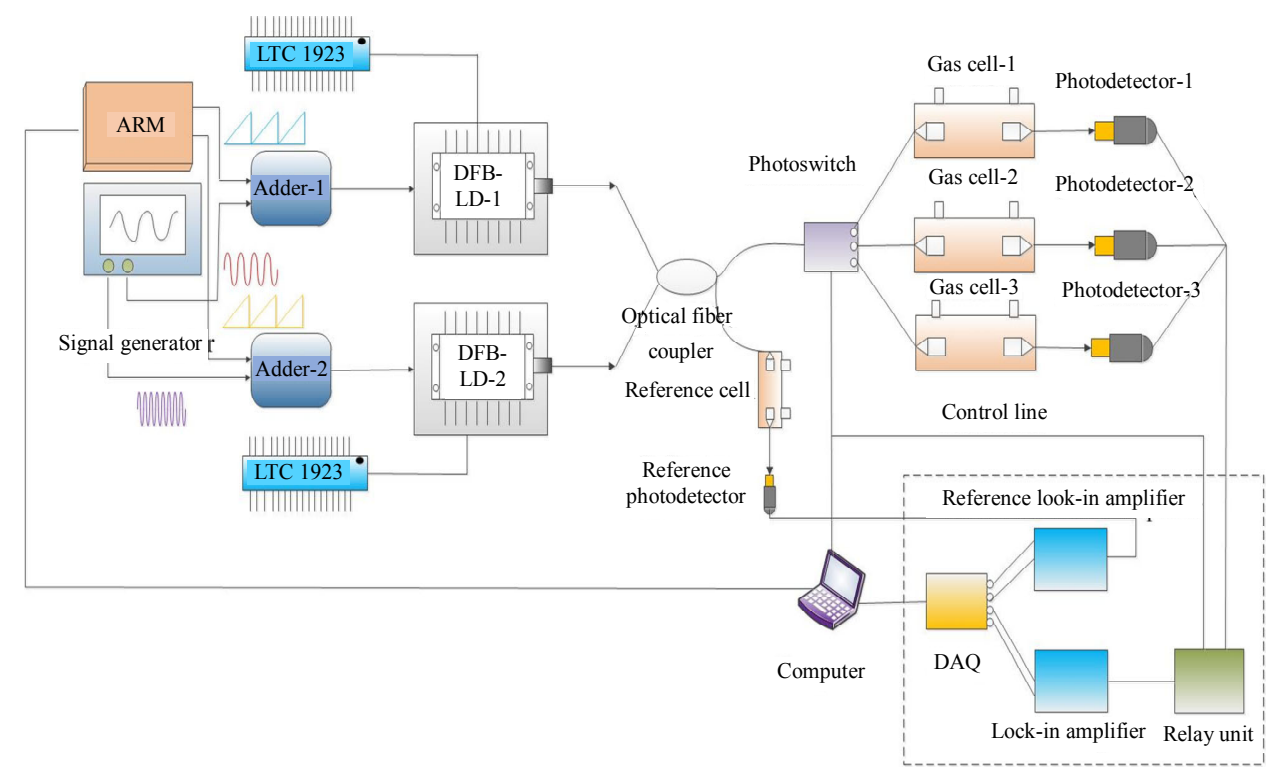

Fig. 4 Experimental setup.

\subsection{Experimental result}

\subsubsection{Multi-component and multi-point detection in WMS}

The experimental setup and the parameters are shown in Fig. 4. A sawtooth wave cycle of $2.4 \mathrm{~s}$ (amplitude: $100 \mathrm{~mA}$ ) and modulation sine waves of $2 \mathrm{kHz}$ and $2.5 \mathrm{kHz}$ (modulation depth: $100 \mathrm{pm}$ ) are chosen to drive the DFB lasers with central wavelengths of $1653.7 \mathrm{~nm}$ and $1368.30 \mathrm{~nm}$ for methane and water vapor detection, respectively. The cross-talk noise between the $2 \mathrm{kHz}$ and $2.5 \mathrm{kHz}$ frequencies is tested, as shown in Fig. 5. As seen in
Fig. 5(a), the $2 f$ signal of $5 \mathrm{kHz}$ is acquired when the modulation sine wave of $2 \mathrm{kHz}$ is the only driver of the DFB laser for methane detection. The cross-talk noise is about $2.01 \mathrm{mV}$ at the $2 \mathrm{kHz}$ modulation frequency. Similarly, the cross-talk noise is about $2.98 \mathrm{mV}$ at the $2.5 \mathrm{kHz}$ modulation frequency, as shown in Fig. 5(b). Our calculation shows that the cross-talk noise can be negligible for methane and water vapor detection.

Detection of methane and water vapor at three points is conducted to verify the feasibility of the multi-component and multi-point trace gas sensing technique using the experimental setup and the 
parameters shown in Fig. 4, at $1600 \mathrm{ppm}$ and $1100 \mathrm{ppm}$, respectively. All the measurements are performed at 1 bar and $24{ }^{\circ} \mathrm{C}$. The photoswitch switching period of $3 \mathrm{~s}$ is used to achieve three-point gas detection. The $2 f$ signals of the three points are shown in detail in Fig. 6. These results demonstrate that multi-component and multi-point detection can be achieved by the proposed scheme using FDM and TDM.
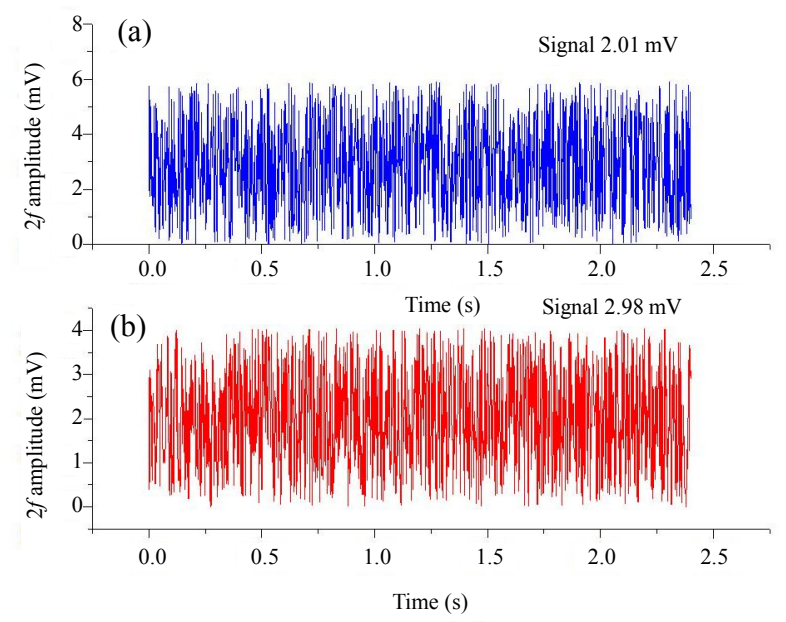

Fig. 5 Cross-talk noise between the frequencies of (a) $2 \mathrm{kHz}$ and (b) $2.5 \mathrm{kHz}$.

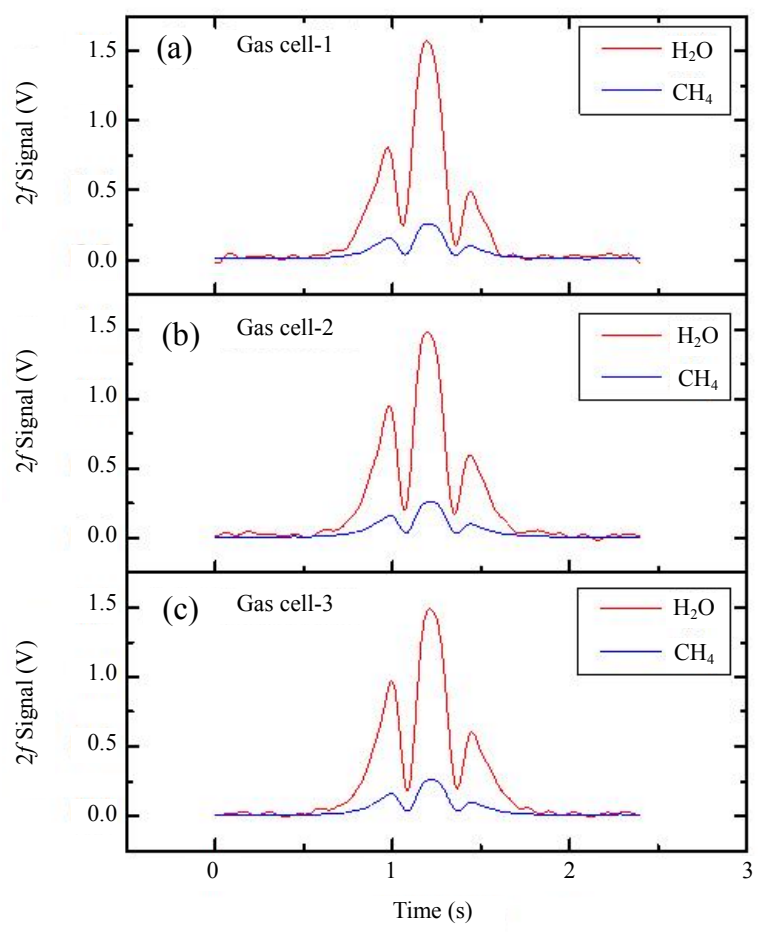

Fig. $62 f$ signal of $\mathrm{H}_{2} \mathrm{O}$ and $\mathrm{CH}_{4}$ at three points: (a) gas cell-1, (b) gas cell-2, and (c) gas cell-3.

\subsubsection{Wavelength stabilization scheme verification experiment}

To verify the effectiveness of the wavelength stabilization scheme, a comparative experiment is conducted using the constant driver current mode in the signal processing method shown in Fig. 3, and the experimental results are shown in Fig. 7. The parameters of the sawtooth wave and the modulation sine wave and the experimental conditions are the same as those mentioned in Section 2.3.1. Figures 7 (a) and 7(b) show the $2 f$ signals of $\mathrm{H}_{2} \mathrm{O}$ and $\mathrm{CH}_{4}$ at $800 \mathrm{ppm}$ and $1600 \mathrm{ppm}$, respectively. In the constant driving mode, the laser driver current is selected as the one at the $2 f$ signal peak, and the current is constant. As seen in Fig. 7, the $2 f$ signal fluctuates over time, whereas it is stable when the wavelength stabilization scheme presented in Section 2.3 is applied. The $2 f$ signal fluctuations of $\mathrm{H}_{2} \mathrm{O}$ and $\mathrm{CH}_{4}$ are about $22 \mathrm{mV}$ and $30 \mathrm{mV}$ with the constant driver current, whereas they are about $6 \mathrm{mV}$ and $7 \mathrm{mV}$ with the wavelength stabilization scheme. Therefore, the proposed scheme is effective for gas absorption center stabilization.
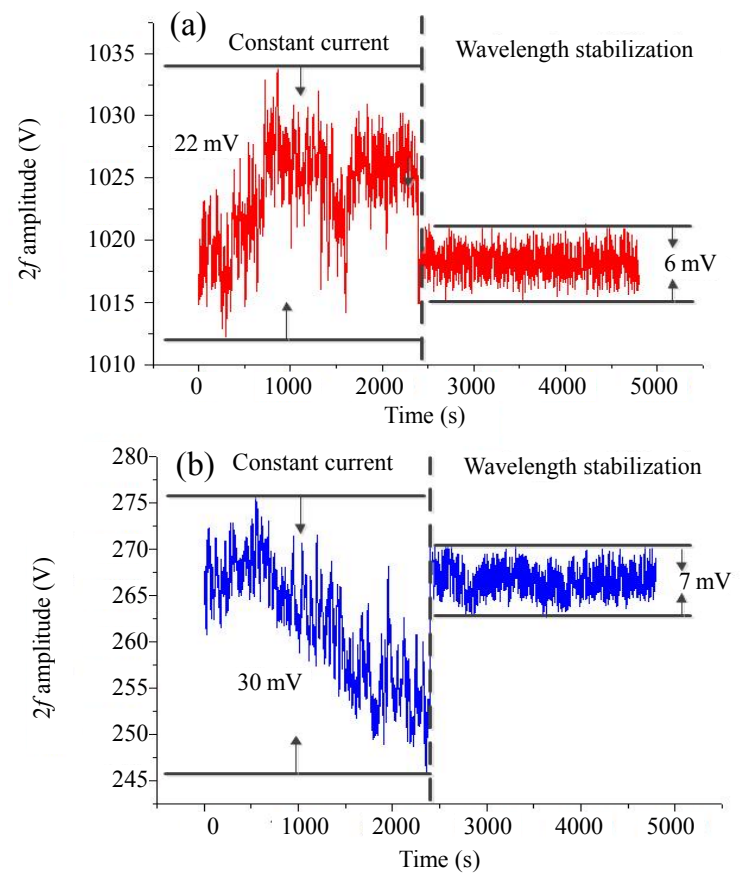

Fig. $72 f$ signal of (a) $\mathrm{H}_{2} \mathrm{O}$ and (b) $\mathrm{CH}_{4}$ in the constant current and wavelength stabilization. 


\subsubsection{System performance with wavelength stabilization scheme}

To demonstrate the improvement of the MDL with the proposed scheme, an experiment to compare the wavelength stabilization scheme with the conventional WMS is conducted, and the experimental setup and parameters are the same as those presented in Section 2.3.1. The improvement of the MDL with the wavelength stabilization scheme is mainly attributed to the narrow lock-in amplifier bandwidth and the numerical averaging algorithm. For the same lock-in amplifier bandwidth of $0.08 \mathrm{~Hz}$, the $2 f$ signals of $\mathrm{H}_{2} \mathrm{O}$ and $\mathrm{CH}_{4}$ at $800 \mathrm{ppm}$ and $1600 \mathrm{ppm}$ with the wavelength stabilization scheme and conventional WMS are shown in Figs. 8 and 9; all of these results are acquired once per $2.4 \mathrm{~s}$. Figure $8(\mathrm{a})$ shows the $2 f$ signals of $\mathrm{H}_{2} \mathrm{O}$ at $800 \mathrm{ppm}$ with the conventional WMS. The figure shows a $364.83 \mathrm{mV}$ signal with $16.84 \mathrm{mV}$ standard deviation for $1200 \mathrm{~s}$ measurement, while a $1103.28 \mathrm{mV}$ signal with $16.31 \mathrm{mV}$ standard deviation for the wavelength stabilization scheme is presented in Fig. 8(b). Figures 9(a) and 9(b) show the $2 f$ signals of $\mathrm{CH}_{4}$ at $1600 \mathrm{ppm}$ with the conventional WMS and the wavelength stabilization scheme, respectively,
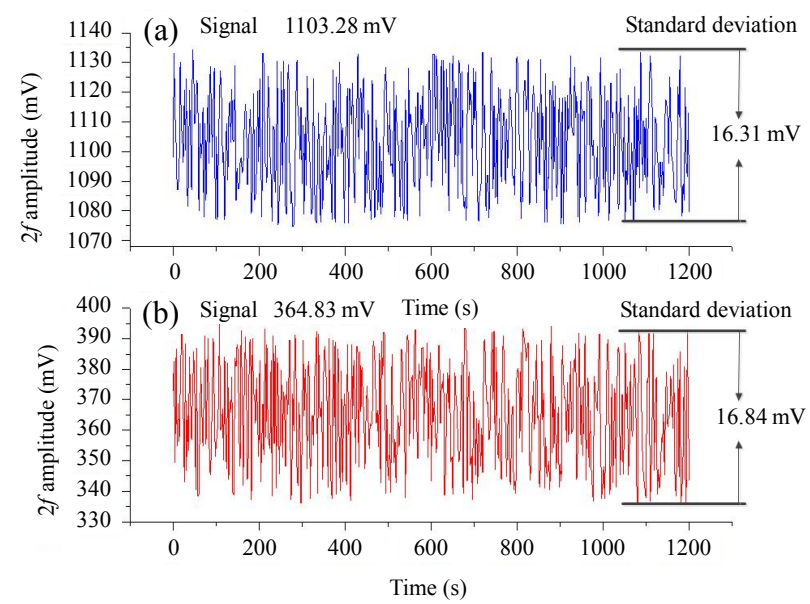

Fig. $82 f$ signal of $\mathrm{H}_{2} \mathrm{O}$ in the (a) conventional WMS and (b) wavelength stabilization scheme with the same lock-in amplifier bandwidth.

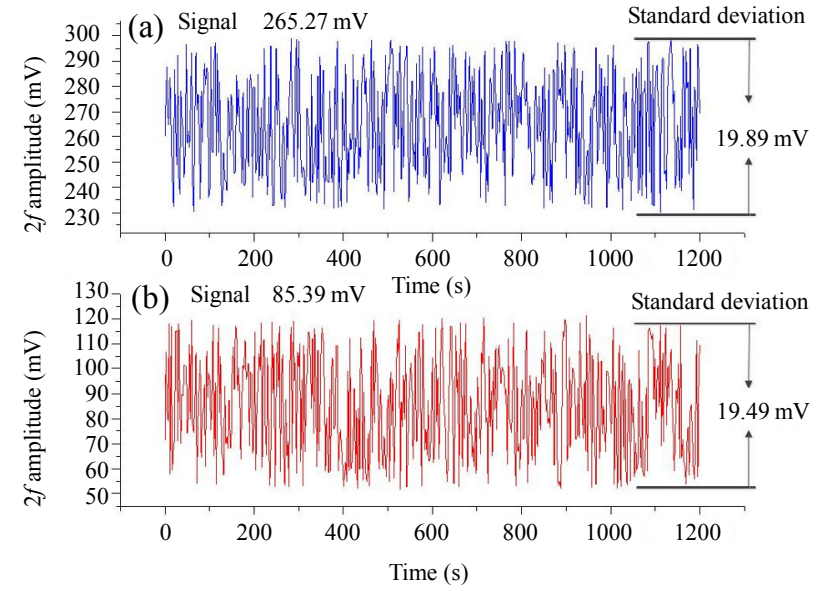

Fig. $92 f$ signal of $\mathrm{CH}_{4}$ in the (a) conventional WMS and (b) wavelength stabilization scheme with the same lock-in amplifier bandwidth.

showing an $85.39 \mathrm{mV}$ signal with $19.49 \mathrm{mV}$ standard deviation and a $265.27 \mathrm{mV}$ signal with $19.89 \mathrm{mV}$ standard deviation. The $2 f$ signals of $\mathrm{H}_{2} \mathrm{O}$ and $\mathrm{CH}_{4}$ with the wavelength stabilization scheme are about 3 times those of the conventional WMS. This may be attributed to the fact that the $2 f$ signals are reduced by the lock-in amplifier bandwidth in the conventional WMS. Therefore, a narrow lock-in amplifier bandwidth can be used in the wavelength stabilization scheme, allowing lower noise in comparison with the conventional WMS.

To conveniently analyze the total MDL improvement with the wavelength stabilization scheme, similar orders of the photoacoustic signal amplitude are achieved with different lock-in amplifier bandwidths. The lock-in amplifier bandwidth in the wavelength stabilization scheme is $0.08 \mathrm{~Hz}$, but for the conventional WMS system, it is $8 \mathrm{~Hz}$. A scanning cycle of $2.4 \mathrm{~s}$ is chosen in WMS, so there would be only one data point of the $2 f$ signal acquired per $2.4 \mathrm{~s}$ in this system. However, in our wavelength stabilization scheme, 6000 data points are collected and averaged by the DAQ in $2.4 \mathrm{~s}$ for a lower noise level. The noise in the wavelength stabilization scheme can be reduced by 
the numerical averaging algorithm. The $2 f$ signals of $\mathrm{H}_{2} \mathrm{O}$ and $\mathrm{CH}_{4}$ at $800 \mathrm{ppm}$ and $1600 \mathrm{ppm}$ in the wavelength stabilization scheme and conventional WMS are shown in Figs. 10 and 11, respectively. As shown in Fig. 10(a), the $2 f$ signal of $\mathrm{H}_{2} \mathrm{O}$ in the conventional WMS is about $1104.59 \mathrm{mV}$ with $16.32 \mathrm{mV}$ standard deviation for $1200 \mathrm{~s}$ measurement, whereas it is about $1100.76 \mathrm{mV}$ with $1.55 \mathrm{mV}$ standard deviation in the wavelength stabilization scheme as shown in Fig. 10(b). The noise in the wavelength stabilization scheme is reduced by the numerical averaging algorithm and narrow lock-in amplifier bandwidth. Our calculations show that the SNR of 710.16 in the wavelength stabilization scheme is 10.5 times higher than that of the conventional WMS. In the wavelength stabilization scheme, the power incident into the gas cell is $5.6 \mathrm{~mW}$, the lock-in amplifier bandwidth is $0.08 \mathrm{~Hz}$, and the gas concentration is $800 \mathrm{ppm}$. Hence, the system normalized noise equivalent absorption coefficient (NNEA) of $1 \sigma$ should be $6.08 \times 10^{-7} \mathrm{~W} \cdot \mathrm{cm}^{-1} \cdot \mathrm{Hz}^{-1 / 2}$, where $\sigma$ is the molecular absorption cross section. With the standard deviation measured to be $1.55 \mathrm{mV}$ for about $1200 \mathrm{~s}$, the MDL is estimated to be $1.13 \mathrm{ppm}$. Moreover, the $2 f$ signals of $\mathrm{CH}_{4}$ and the standard deviation in the conventional WMS and the wavelength stabilization scheme are $264.02 \mathrm{mV}$, $19.66 \mathrm{mV}, 261.92 \mathrm{mV}$, and $1.94 \mathrm{mV}$, respectively, as shown in Figs. 11(a) and 11(b). The SNR of 135.01 in the wavelength stabilization scheme is also 10.1 times higher than that of the conventional WMS, and the MDL is estimated to be $11.85 \mathrm{ppm}$. Because the power incident into the gas cell is $7.6 \mathrm{~mW}$, the lock-in amplifier bandwidth is $0.08 \mathrm{~Hz}$, and the gas concentration is $1600 \mathrm{ppm}$, the NNEA of $1 \sigma$ should be $7.8 \times 10^{-7} \mathrm{~W} \cdot \mathrm{cm}^{-1} \cdot \mathrm{Hz}^{-1 / 2}$.

Finally, the sensor linearity is examined by measuring a series of $\mathrm{H}_{2} \mathrm{O}$ and $\mathrm{CH}_{4}$ concentrations
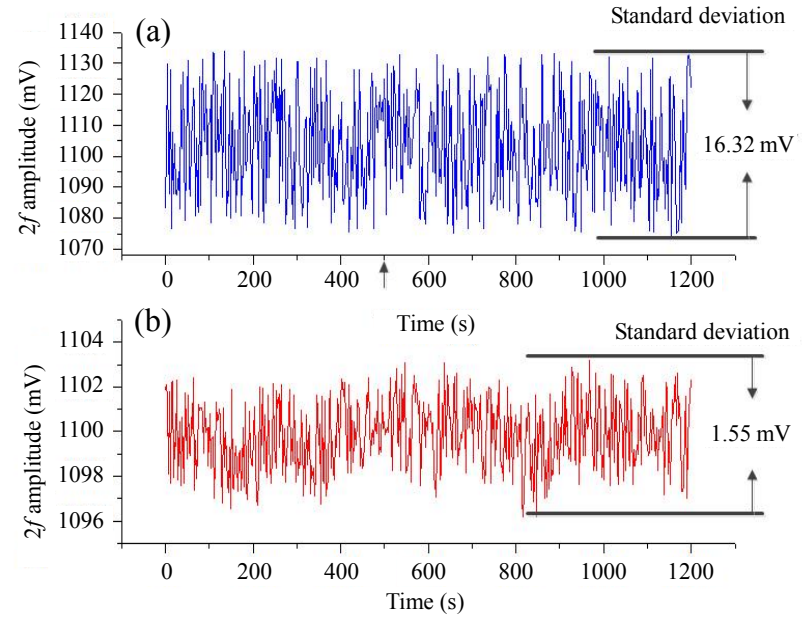

Fig. $102 f$ signal of $\mathrm{H}_{2} \mathrm{O}$ in the (a) conventional WMS and (b) wavelength stabilization scheme with different lock-in amplifier bandwidths.

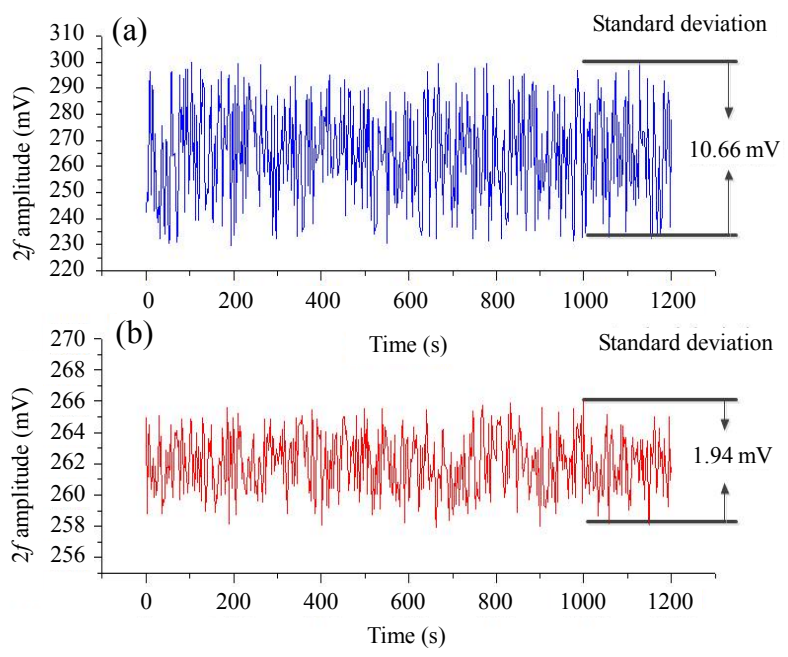

Fig. $112 f$ signal of $\mathrm{CH}_{4}$ in the (a) conventional WMS and (b) wavelength stabilization scheme with different lock-in amplifier bandwidths.

by using the wavelength stabilization scheme. The obtained $2 f$ signals at various gas concentrations ranging from $500 \mathrm{ppm}$ to $3000 \mathrm{ppm}$ are plotted in Figs. 12 and 13. The $2 f$ PAS signals of $\mathrm{H}_{2} \mathrm{O}$ and $\mathrm{CH}_{4}$ are also plotted in Figs. 14 and 15 as a function of gas concentration. The linear fit of the obtained data yields the $R$-square values of 0.9983 and 0.99564 , indicating the excellent linear responses of the sensor to $\mathrm{H}_{2} \mathrm{O}$ and $\mathrm{CH}_{4}$ concentrations, respectively. 


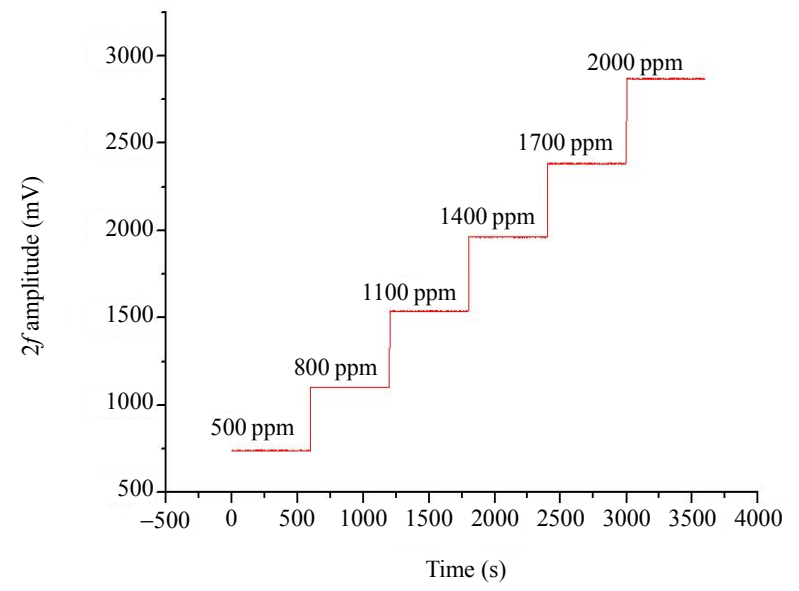

Fig. $122 f$ signal of $\mathrm{H}_{2} \mathrm{O}$ in the wavelength stabilization scheme.

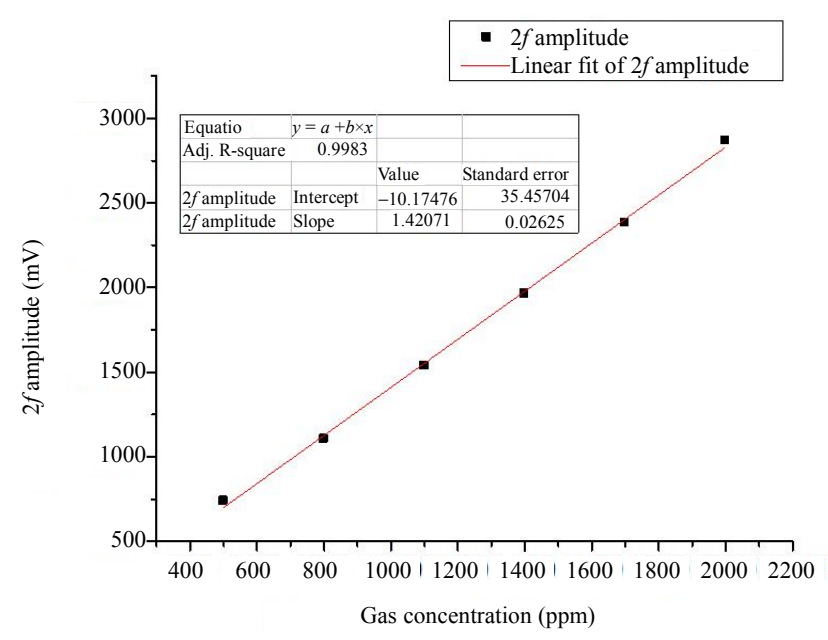

Fig. $142 f$ signal linearity of $\mathrm{H}_{2} \mathrm{O}$ in the wavelength stabilization scheme.

\section{Discussion}

With advances in science and technology, the application field of gas detection is expanding, and the number of gas detection sensors is also increasing. To reduce the examination costs, multi-point and multi-component gas sensors have been intensively studied and have attracted much attention. Most of the current gas sensors only have a single function, namely, either multi-component or multi-point gas detection. Very few sensors have both functions. In this paper, multi-component and multi-point trace gas sensing in WMS is demonstrated based on FDM and TDM. A reference

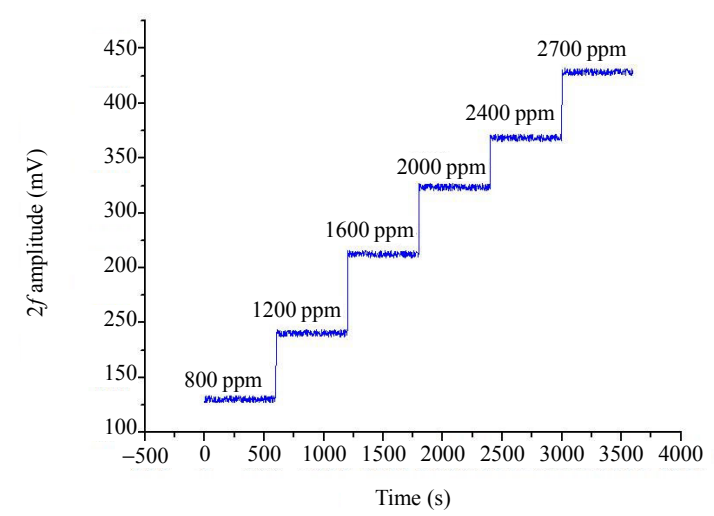

Fig. $132 f$ signal of $\mathrm{CH}_{4}$ in the wavelength stabilization scheme.

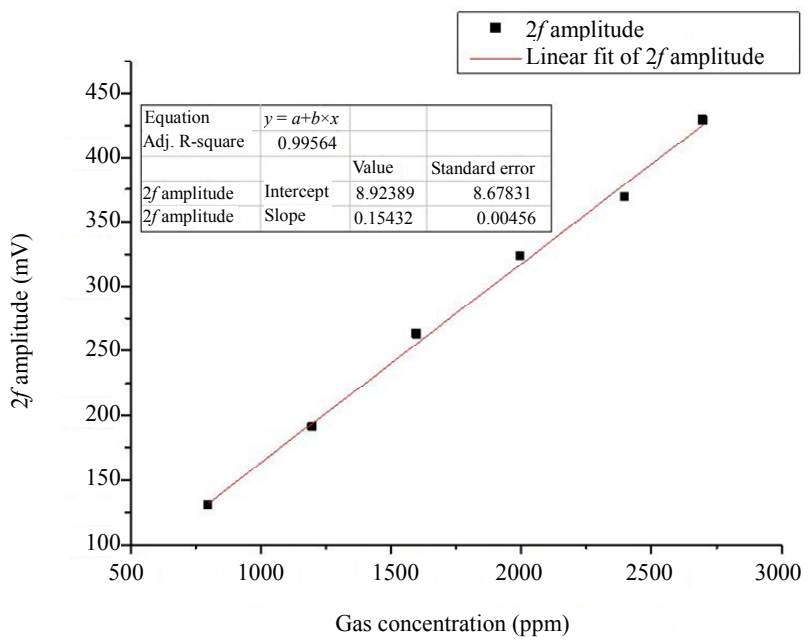

Fig. $152 f$ signal linearity of $\mathrm{CH}_{4}$ in the wavelength stabilization scheme.

photodetector and a gas cell with the constant concentration are connected in series to measure the $2 f$ signal peak of the components for wavelength stabilization in real time. The central wavelengths of the DFB lasers are locked to the target gas absorption centers by the $2 f$ signal peak of the reference gas cell by using a digital PID controller. This wavelength stabilization scheme allows an averaging algorithm to improve the MDL. Trace gas sensing of two-components, $\mathrm{H}_{2} \mathrm{O}$ and $\mathrm{CH}_{4}$, at three points in a measured gas cell is conducted to illustrate the effectiveness of this scheme. 


\section{Conclusions}

In this paper, multi-component and multi-point trace gas sensing in WMS is proposed based on FDM and TDM; the MDL is significantly improved by the application of the wavelength stabilization scheme. A reference photodetector and a gas cell with constant concentration are used to achieve wavelength stabilization in real time based on the reference $2 f$ signal peak. The $2 f$ signal peak detected by the reference LIA is processed with a set value using a digital PID controller to lock the target gas absorption center in real time. Two-component and three-point trace gas sensing is taken as an example to demonstrate the multi-component and multi-point trace gas sensing proposed in this paper. Two DFB lasers with $1650.9 \mathrm{~nm}$ and $1368.597 \mathrm{~nm}$ as well as $2 \mathrm{kHz}$ and $2.5 \mathrm{kHz}$ modulation frequencies are injected into the gas cell to achieve $\mathrm{H}_{2} \mathrm{O}$ and $\mathrm{CH}_{4}$ measurement, and three-point trace gas sensing is achieved by the photoswitch and relay unit. The comparative experiments show that the MDLs of the two components are $1.13 \mathrm{ppm}\left(\mathrm{H}_{2} \mathrm{O}\right)$ and $11.85 \mathrm{ppm}$ $\left(\mathrm{CH}_{4}\right)$, respectively, which is an improvement of 10.5 and 10.1 times in comparison with the traditional WMS. Meanwhile, their excellent $R$-square values reach 0.9983 and 0.99564 for the concentration ranges of $500 \mathrm{ppm}$ to $2000 \mathrm{ppm}$ and $800 \mathrm{ppm}$ to $2700 \mathrm{ppm}$, respectively.

\section{Acknowledgment}

This work was supported by the Research Fund for the Doctoral Program of Liao Cheng University (Grant No. 318051543) and the National Natural Science Foundation of China (Grant No. 61475085).

Open Access This article is distributed under the terms of the Creative Commons Attribution 4.0 International License (http://creativecommons.org/licenses/by/4.0/), which permits unrestricted use, distribution, and reproduction in any medium, provided you give appropriate credit to the original author(s) and the source, provide a link to the Creative Commons license, and indicate if changes were made.

\section{References}

[1] S. Rasi, A. Veijanen, and J. Rintala, "Trace compounds of biogas from different biogas production plants," Energy, 2007, 32(8): 1375-1380.

[2] X. Chen, J. Chang, F. P. Wang, Z. L. Wang, W. Wei, Y. Y. Liu, et al., "A portable analog lock-in amplifier for accurate phase measurement and application in high-precision optical oxygen concentration detection," Photonic Sensors, 2017, 7(1): 27-36.

[3] X. G. Niu, X. Huang, Z. Zhao, Y. H. Zhang, C. C. Huang, and L. Cui, "The design and evaluation of a wireless sensor network for mine safety monitoring," in Proceeding of IEEE Global Telecommunications Conference, Washington, DC, USA, 2007, pp. 1291-1295.

[4] J. P. SUN, "Mine safety monitoring and control technology and system," Coal Science and Technology, 2010, 38(10): 1-4.

[5] M. G. Allen, "Diode laser absorption sensors for gas-dynamic and combustion flows," Measurement Science and Technology, 1998, 9(4): 545-562.

[6] Y. Liu, E. Koep, and M. L. Liu, "A highly sensitive and fast-responding $\mathrm{SnO}_{2}$ sensor fabricated by combustion chemical vapor deposition," Chemistry of Materials, 2005, 17(15): 3997-4000.

[7] Q. D. Zhang, J. Chang, Z. L. Wang, F. P. Wang, F. T. Jiang, and M. Y. Wang, "SNR improvement of QEPAS system by preamplifier circuit optimization and frequency locked technique," Photonic Sensors, 2018, 8(2): 127-133.

[8] Z. R. Zhang, T. Pang, Y. Yang, H. Xia, X. J. Cui, P. S. Sun, et al., "Development of a tunable diode laser absorption sensor for online monitoring of industrial gas total emissions based on optical scintillation cross-correlation technique," Optics Express, 2016, 24(10): A943-A955.

[9] L. Dong, F. K. Tittel, C. Li, N. P. Sanchez, H. Wu, C. Zheng, et al., "Compact TDLAS based sensor design using interband cascade lasers for mid-ir trace gas sensing," Optics Express, 2016, 24(6): A528-A535.

[10] K. Sun, X. Chao, R. Sur, C. S. Goldenstein, J. B. Jeffries, and R. K. Hanson, "Analysis of calibration-free wavelength-scanned wavelength modulation spectroscopy for practical gas sensing using tunable diode lasers," Measurement Science \& Technology, 2013, 24(12): 5203-338.

[11] B. Tao, Z. Y. Hu, W. Fan, S. Wang, J. F. Ye, and Z. R. Zhang, "Novel method for quantitative and real-time measurements on engine combustion at varying pressure based on the wavelength modulation spectroscopy," Optics Express, 2017, 25(16): A762-A776.

[12] G. Stewart, J. R. P. Bain, K. Ruxton, K. Duffin, M. Lengden, and W. Johnstone, "Recovery of absolute gas absorption line shapes using tunable diode laser spectroscopy with wavelength modulation-part 2 : 
experimental investigation," Journal of Lightwave Technology, 2011, 29(7): 987-996.

[13] S. Eich, E. Schmälzlin, and H. G. Löhmannsröben, "Distributed fiber optical sensing of molecular oxygen with OTDR," SPIE, 2010, 7726: 77260A-1-77260A-8.

[14] S. Eich, E. Schmälzlin, and H. G. Löhmannsröben, "Distributed fiber optical sensing of oxygen with optical time domain reflectometry," Sensors, 2013, 13: 7170-7183.

[15] C. Sun, Y. P. Chen, G. Zhang, F. Wang, G. S. Liu, and J. J. Ding, "Multipoint remote methane measurement system based on spectrum absorption and reflective TDM," IEEE Photonic Technology Letters, 2016, 28: 2487-2490.

[16] Z. H. Liu, Y. Wei, Y. Zhang, Y. S. Wang, E. M. Zhao, Y. X. Zhang, et al., "A multi-channel fiber SPR sensor based on TDM technology," Sensors And Actuators B: Chemical, 2016, 226: 326-331.

[17] Y. He, Y. F. Ma, Y. Tong, X. Yu, Z. F. Peng, J. Gao, et al., "Long distance, distributed gas sensing based on micro-nano fiber evanescent wave quartz-enhanced photoacoustic spectroscopy," Applied Physics Letters, 2017, 111(24): 241102-1-241102-4.

[18] L. Yu, T. Liu, K. Liu, J. Jiang, and T. Wang, "Intracavity multigas detection based on multiband fiber ring laser," Sensors \& Actuators B: Chemical, 2016, 226: 170-175.

[19] H. Wu, L. Dong, X. Liu, H. D. Zheng, X. K. Yin, W. G. Ma, et al., "Fiber-amplifier-enhanced QEPAS sensor for simultaneous trace gas detection of $\mathrm{NH}_{3}$ and $\mathrm{H}_{2} \mathrm{~S}$," Sensors, 2015, 15(10): 26743-26755.

[20] J. J. Scherer, J. B. Paul, H. J. Jost, and M. L. Fischer, "Mid-IR difference frequency laser-based sensors for ambient $\mathrm{CH}_{4}, \mathrm{CO}$, and $\mathrm{N}_{2} \mathrm{O}$ monitoring," Applied Physics B, 2013, 110(2): 271-277.

[21] M. Jahjah, W. Ren, P. Stefański, R. Lewicki, J. W. Zhang, and W. Z. Jiang, "A compact QCL based methane and nitrous oxide sensor for environmental and medical applications," Analyst, 2014, 139(9): 2065-2069.

[22] Y. Ma, R. Lewicki, M. Razeghi, and F. K. Tittel, "QEPAS based ppb-level detection of $\mathrm{CO}$ and $\mathrm{N}_{2} \mathrm{O}$ using a high power CW DFB-QCL," Optics Express, 2013, 21(1): 1008-1019.

[23] Y. Zhang, M. Zhang, and W. Jin, "Multipoint, fiber-optic gas detection with intra-cavity spectroscopy," Optics Communications, 2003, 220(4): 361-364.

[24] F. Ye, L. Qian, and B. Qi, "Multipoint chemical gas sensing using frequency-shifted interferometry," Journal of Lightwave Technology, 2009, 27(23): 5356-5364.

[25] G. Whitenett, G. Stewart, H. Yu, and B. Culshaw, "Investigation of a tuneable mode-locked fiber laser for application to multipoint gas spectroscopy," Journal of Lightwave Technology, 2004, 22(3): 813-819.

[26] Q. Wang, Z. Wang, and W. Ren, "Wavelength-stabilization-based photoacoustic spectroscopy for methane detection," Measurement Science and Technology, 2017, 28(6): 065102-1-065102-7. 